\begin{tabular}{lc}
\hline & Sharif University of Technology \\
SCIENTIA & Transactions B: Mechanical Engineering \\
IRAN ICA & www.scientiairanica.com \\
\hline
\end{tabular}

\title{
First-order optimal line-of-sight guidance for stationary targets
}

\author{
S.H. Jalali-Naini* and S.H. Sajjadi \\ Faculty of Mechanical Engineering, Tarbiat Modares University, Tehran, P. O. Box 14115-111, Iran.
}

Received 24 April 2014; received in revised form 10 February 2015; accepted 4 May 2015

\section{KEYWORDS}

Line-of-sight guidance; Optimal guidance; Normalized miss distance analysis; First order control system.

\begin{abstract}
In this paper, a closed-loop optimal line-of-sight guidance law for first-order control systems is derived for stationary targets. The problem is solved for the onedimensional case using normalized equations to obtain normalized guidance gains and performance curves. Three sets of normalized equations are introduced and discussed using different normalizing factors. The performances of the guidance laws are compared in normalized forms with zero-lag optimal guidance and a first-order optimal scheme with steady-state gains using a second-order control system. Normalized miss distance analysis shows that the miss distance of the first-order guidance law is smaller than the two mentioned schemes for small total flight times.
\end{abstract}

(C) 2016 Sharif University of Technology. All rights reserved.

\section{Introduction}

Guidance laws are categorized into two-point and three-point guidance schemes [1]. The three-point guidance is sometimes referred to as Line-Of-Sight (LOS) guidance, in which the vehicle maneuvers to keep itself on the line between a reference point and a target point, i.e. reference-to-target line-of-sight $[1,2]$. This type of guidance law is used in the trajectory design of moving vehicles, especially aerial vehicles, such as missiles [2,3] and unmanned aircraft. LOS guidance can be considered a special case for trajectory tracking and terrain following. In other words, the three-point guidance scheme can be extended when the reference line is not straight, e.g. in a terrain following problem $[4,5]$.

Modern control theory and estimation are utilized to develop three-point guidance laws and trajectory tracking control strategies, such as feedback lineariza-

*. Corresponding author. Tel.: +98 21 82883936; Fax: +982182884909

E-mail addresses: shjalalinaini@modares.ac.ir (S.H. Jalali-Naini); h.sajjadi@modares.ac.ir (S.H. Sajjadi) tion [6], fuzzy-logic [7-9], robust [10], adaptive [11], variable-structure [12], linear model predictive control [13] and optimal control [14-16]. The genetic algorithm [17], ant colony optimization [18], and neural network [19] may also be used in the design and development of this class of guidance and control laws.

These control methods are also used in two-point guidance schemes. For example, two-point Optimal Guidance Laws (OGLs) in closed-loop are available for first-, second-, and high-order control systems [20]. Analytical approaches for three-point guidance are more difficult than two-point guidance, because of the additional constraint on the vehicle trajectory for keeping it near the reference-to-target LOS. Only simple cases are solved analytically in closed-form for three-point guidance, e.g. as treated in $[21,22]$ for a case of idealized LOS trajectory.

Derivation of optimal three-point guidance laws is also more complex than the two-point strategies, because of the above-mentioned additional constraint on vehicle trajectory. The general formulation of the optimal LOS guidance, deterministic [23] or stochastic [23,24] approach for linear systems can be derived simply from linear optimal control theory. However, 
the solutions of the resulting relations in closed-form are of most interest in guidance theory and application. The optimal solutions for perfect control system of linearized LOS problem have been obtained in $[14,15]$ for stationary targets; however, their performance indices are slightly different. The guidance gains of [15] have also been derived for a steady-state solution. Increasing the order of the control system highly increases solution difficulties in the closed-form. The optimal solution of the first-order system has been presented in [25] for specified values of parameters, without obtaining explicit relations for guidance gains in terms of time constant, time-to-go, and LOS weighting factor.

In this paper, the optimal solution of LOS guidance is derived for a first-order control system and guidance gains are obtained explicitly in terms of time constant, time-to-go until the final time, and LOS weighting factor. The relations are also normalized to give more insight into the design and performance analysis of the guidance law.

The rest of the paper is organized as follows: Section 2 presents the governing equations of motion in normalized form. Section 3 formulates the optimal lineof-sight guidance in closed-form. Section 4 presents the simulation results and comparison of the proposed first-order OGL with other guidance laws, followed by conclusions in Section 5.

\section{Normalized equations of motion}

The governing equation of motion of a vehicle, $P$, as a point mass for a one-dimensional case is $\ddot{h}=a$, where $h$ is the distance of the vehicle from the line between reference point $\mathrm{O}$ and the target position, as shown in Figure 1. Also, $a$ is the vehicle acceleration assumed in the direction normal to the reference-totarget LOS. The overdot indicates differentiation with respect to time. The control system is taken as a firstorder transfer function from commanded acceleration $u$ to achieved commanded acceleration $a$, that is:

$$
\frac{a}{u}(s)=\frac{1}{1+T s}
$$

where $T$ is the time constant of the control system. The

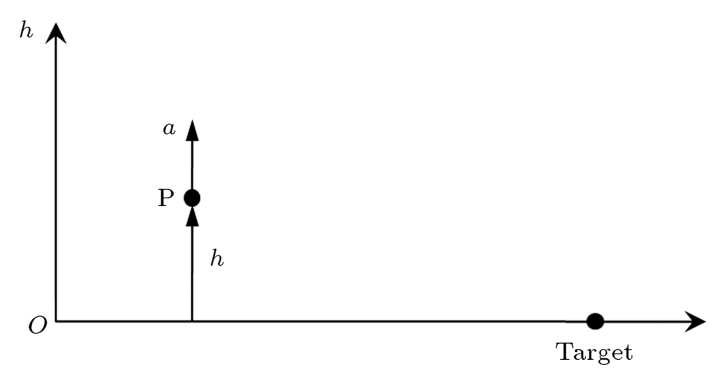

Figure 1. Geometry of one-dimensional problem. state space representation of the problem is given by:

$$
\left\{\begin{array}{l}
\dot{h}=v \\
\dot{v}=a \\
\dot{a}=(u-a) / T
\end{array}\right.
$$

where $v$ is the velocity component of vehicle $\mathrm{P}$ normal to LOS (along the $h$ axis in Figure 1). The state space equations are normalized using the following change of variables:

$$
\begin{aligned}
\tau & =\frac{t}{T}, & \tau_{f}=\frac{t_{f}}{T}, & \tau_{g o}=\frac{t_{g o}}{T}, \\
\hat{h} & =\frac{h}{A T^{2}}, & \hat{v} & =\frac{v}{A T}, \\
\hat{a} & =\frac{a}{A}, & \hat{u} & =\frac{u}{A},
\end{aligned}
$$

where $A$ is a normalizing parameter in $\mathrm{m} / \mathrm{s}^{2}$. Here, the normalizing factor in [26] is generalized using parameter $A$. Also, $t_{f}$ is the final time and $t_{g o}=t_{f}-t$ is the time-to-go until the final time. The normalized state space form is then given by:

$$
\left\{\begin{array}{l}
\hat{h}^{\prime}=\hat{v} \\
\hat{v}^{\prime}=\hat{a} \\
\hat{a}^{\prime}=\hat{u}-\hat{a}
\end{array}\right.
$$

where ()$^{\prime}$ denotes differentiation with respect to the nondimensional time, $\tau$.

When the control system is given in the form of:

$$
\frac{a}{u}(s)=\frac{1}{\left(1+\frac{T}{2} s\right)^{2}},
$$

similar to our simulation, the last equation of the state equations is replaced by the two following equations:

$$
\left\{\begin{array}{l}
\dot{x}_{1}=\frac{2}{T}\left(u-x_{1}\right) \\
\dot{a}=\frac{2}{T}\left(x_{1}-a\right)
\end{array}\right.
$$

or, in the normalized form, as:

$$
\left\{\begin{array}{l}
\hat{x}_{1}=2\left(\hat{u}-\hat{x}_{1}\right) \\
\hat{a}^{\prime}=2\left(\hat{x}_{1}-\hat{a}\right)
\end{array}\right.
$$

where $x_{1}$ is the additional state variable due to increasing the order of the control system, and $\hat{x}_{1}=x_{1} / A$.

The second-order control system (Eq. (7)) described by the preceding relations is used for the performance analysis of LOS guidance laws in the implemented simulation code. Since the first-order OGL is obtained assuming a first-order control system, the performance analysis is carried out for the higherorder control system; otherwise the comparison will not be justified. 


\section{Optimal guidance problem}

The problem is to find the commanded acceleration, $u$, as the input control that minimizes the performance index:

$$
J=\frac{1}{2} \int_{t_{0}}^{t_{f}}\left(b h^{2}+u^{2}\right) d t,
$$

subject to state space Eq. (2), where $b>0$ is the weighting coefficient for the vehicle deviation from the LOS. The initial and final conditions are:

$$
\left\{\begin{array} { l } 
{ h ( t _ { 0 } ) = h _ { 0 } } \\
{ v ( t _ { 0 } ) = v _ { 0 } } \\
{ a ( t _ { 0 } ) = a _ { 0 } }
\end{array} \quad \left\{\begin{array}{l}
h\left(t_{f}\right)=0 \\
v\left(t_{f}\right)=\text { free } \\
a\left(t_{f}\right)=\text { free }
\end{array}\right.\right.
$$

where subscript " 0 " indicates the initial value, and the final time, $t_{f}$, is specified.

The problem is now restated in the normalized form to obtain the normalized commanded acceleration, $\hat{u}$, that minimizes the following performance index:

$$
\frac{J}{T A^{2}}=\frac{1}{2} \int_{0}^{\tau_{f}}\left(\hat{b} \hat{h}^{2}+\hat{u}^{2}\right) d \tau, \quad \hat{b}=b T^{4},
$$

subject to the normalized state space Eq. (6). The nondimensional initial and final conditions are:

$$
\left\{\begin{array} { l } 
{ \hat { h } ( \tau _ { 0 } ) = \hat { h } _ { 0 } } \\
{ \hat { v } ( \tau _ { 0 } ) = \hat { v } _ { 0 } } \\
{ \hat { a } ( \tau _ { 0 } ) = \hat { a } _ { 0 } }
\end{array} \quad \left\{\begin{array}{l}
\hat{h}\left(\tau_{f}\right)=0 \\
\hat{v}\left(\tau_{f}\right)=\text { free } \\
\hat{a}\left(\tau_{f}\right)=\text { free }
\end{array}\right.\right.
$$

The final nondimensional time, $\tau_{f}$, is also specified. The Hamiltonian of the normalized problem is given by:

$$
H=\frac{1}{2} \hat{b} \hat{h}^{2}+\frac{1}{2} \hat{u}^{2}+\lambda_{h} \hat{v}+\lambda_{v} \hat{a}+\lambda_{a}(\hat{u}-\hat{a}) .
$$

The optimality condition gives:

$$
\frac{\partial H}{\partial \hat{u}}=0 \rightarrow \hat{u}=-\lambda_{a} .
$$

Costate vector $\vec{\lambda}$ is simply obtained as:

$$
\dot{\vec{\lambda}}=-\frac{\partial H}{\partial \vec{x}} \rightarrow\left\{\begin{array}{l}
\dot{\lambda}_{h}=-\hat{b} \hat{h} \\
\dot{\lambda}_{v}=-\lambda_{h} \\
\dot{\lambda}_{a}=-\lambda_{v}+\lambda_{a}
\end{array}\right.
$$

where $\vec{x}=\left[\begin{array}{lll}\hat{h} & \hat{v} & \hat{a}\end{array}\right]^{T}$ and $\vec{\lambda}=\left[\begin{array}{lll}\lambda_{h} & \lambda_{v} & \lambda_{a}\end{array}\right]^{T}$. Since the final values for $\hat{v}\left(\tau_{f}\right)$ and $\hat{a}\left(\tau_{f}\right)$ are free, we have:

$$
\left\{\begin{array}{l}
\lambda_{v}\left(\tau_{f}\right)=0 \\
\lambda_{a}\left(\tau_{f}\right)=0
\end{array}\right.
$$

To solve the problem, the state and costate equations are combined as follows [27]:

$$
\frac{d}{d \tau}\left[\begin{array}{l}
\vec{x} \\
\vec{\lambda}
\end{array}\right]=A_{p}\left[\begin{array}{l}
\vec{x} \\
\vec{\lambda}
\end{array}\right],
$$

where:

$$
A_{P}=\left[\begin{array}{cccccc}
0 & 1 & 0 & 0 & 0 & 0 \\
0 & 0 & 1 & 0 & 0 & 0 \\
0 & 0 & -1 & 0 & 0 & -1 \\
-\hat{b} & 0 & 0 & 0 & 0 & 0 \\
0 & 0 & 0 & -1 & 0 & 0 \\
0 & 0 & 0 & 0 & -1 & 1
\end{array}\right] .
$$

From Eqs. (13) and (17), the nondimensional initial and final conditions are:

$$
\left\{\begin{array} { l } 
{ \hat { h } ( \tau _ { 0 } ) = \hat { h } _ { 0 } } \\
{ \hat { v } ( \tau _ { 0 } ) = \hat { v } _ { 0 } } \\
{ \hat { a } ( \tau _ { 0 } ) = 0 }
\end{array} \quad \left\{\begin{array}{l}
\hat{h}\left(\tau_{f}\right)=0 \\
\lambda_{v}\left(\tau_{f}\right)=0 \\
\lambda_{a}\left(\tau_{f}\right)=0
\end{array}\right.\right.
$$

The solution of state space equation (18) in terms of the initial conditions has the form:

$$
\left[\begin{array}{l}
\vec{x}(\tau) \\
\vec{\lambda}(\tau)
\end{array}\right]_{6 \times 1}=\Phi\left(\tau-\tau_{0}\right)\left[\begin{array}{l}
\vec{x}\left(\tau_{0}\right) \\
\vec{\lambda}\left(\tau_{0}\right)
\end{array}\right]_{6 \times 1},
$$

where $\Phi$ is the state transition matrix for the system matrix, $A_{p}$. Therefore, we have:

$$
\Phi\left(\tau-\tau_{0}\right)=\mathcal{L}^{-1}\left\{\left(s I-A_{p}\right)^{-1}\right\}_{\tau-\tau_{0}},
$$

where $I$ is a $6 \times 6$ identity matrix, $s$ is the Laplace transform variable, and $\mathcal{L}^{-1}$ denotes the inverse Laplace transform. The state transition matrix elements can be solved analytically for the problem (see Appendix A). Eq. (21) may be written from the current nondimensional time, $\tau$, to $\tau_{f}$, as follows:

$$
\left[\begin{array}{l}
\vec{x}\left(\tau_{f}\right) \\
\vec{\lambda}\left(\tau_{f}\right)
\end{array}\right]_{6 \times 1}=\Phi\left(\tau_{g o}\right)\left[\begin{array}{l}
\vec{x}(\tau) \\
\vec{\lambda}(\tau)
\end{array}\right]_{6 \times 1} .
$$

Applying the final conditions, $\hat{h}\left(\tau_{f}\right)=0, \lambda_{v}\left(\tau_{f}\right)=0$, and $\lambda_{a}\left(\tau_{f}\right)=0$, for the first, fifth, and sixth rows of the preceding relation, yields:

$$
\begin{gathered}
\phi_{11}\left(\tau_{g o}\right) \hat{h}(\tau)+\phi_{12}\left(\tau_{g o}\right) \hat{v}(\tau)+\phi_{13}\left(\tau_{g o}\right) \hat{a}(\tau) \\
+\phi_{14}\left(\tau_{g o}\right) \lambda_{h}(\tau)+\phi_{15} \lambda_{v}(\tau)+\phi_{16} \lambda_{a}(\tau)=0, \\
\phi_{51}\left(\tau_{g o}\right) \hat{h}(\tau)+\phi_{52}\left(\tau_{g o}\right) \hat{v}(\tau)+\phi_{53}\left(\tau_{g o}\right) \hat{a}(\tau) \\
\quad+\phi_{54}\left(\tau_{g o}\right) \lambda_{h}(\tau)+\phi_{55} \lambda_{v}(\tau)+\phi_{56} \lambda_{a}(\tau)=0, \\
\phi_{61}\left(\tau_{g o}\right) \hat{h}(\tau)+\phi_{62}\left(\tau_{g o}\right) \hat{v}(\tau)+\phi_{63}\left(\tau_{g o}\right) \hat{a}(\tau) \\
\quad+\phi_{64}\left(\tau_{g o}\right) \lambda_{h}(\tau)+\phi_{65} \lambda_{v}(\tau)+\phi_{66} \lambda_{a}(\tau)=0 .
\end{gathered}
$$


The three preceding equations are rewritten in the following matrix form:

$$
P_{1}\left(\tau_{g o}\right) \vec{x}(\tau)+P_{2}\left(\tau_{g o}\right) \vec{\lambda}(\tau)=\overrightarrow{0}
$$

where:

$$
\begin{aligned}
& P_{1}\left(\tau_{g o}\right)=\left[\begin{array}{lll}
\phi_{11}\left(\tau_{g o}\right) & \phi_{12}\left(\tau_{g o}\right) & \phi_{13}\left(\tau_{g o}\right) \\
\phi_{51}\left(\tau_{g o}\right) & \phi_{52}\left(\tau_{g o}\right) & \phi_{53}\left(\tau_{g o}\right) \\
\phi_{61}\left(\tau_{g o}\right) & \phi_{62}\left(\tau_{g o}\right) & \phi_{63}\left(\tau_{g o}\right)
\end{array}\right], \\
& P_{2}\left(\tau_{g o}\right)=\left[\begin{array}{lll}
\phi_{14}\left(\tau_{g o}\right) & \phi_{15}\left(\tau_{g o}\right) & \phi_{16}\left(\tau_{g o}\right) \\
\phi_{54}\left(\tau_{g o}\right) & \phi_{55}\left(\tau_{g o}\right) & \phi_{56}\left(\tau_{g o}\right) \\
\phi_{64}\left(\tau_{g o}\right) & \phi_{65}\left(\tau_{g o}\right) & \phi_{66}\left(\tau_{g o}\right)
\end{array}\right] .
\end{aligned}
$$

If $P_{2}\left(\tau_{g o}\right)$ is invertible, $\vec{\lambda}(\tau)$ can be obtained from Eq. (24) as:

$$
\vec{\lambda}(\tau)=-P_{2}\left(\tau_{g o}\right)^{-1} P_{1}\left(\tau_{g o}\right) \vec{x}(\tau) .
$$

Costate $\lambda_{a}$, from the preceding relation, is written as follows:

$$
\lambda_{a}(\tau)=\hat{C}_{h}\left(\tau_{g o}\right) \hat{h}+\hat{C}_{v}\left(\tau_{g o}\right) \hat{v}+\hat{C}_{a}\left(\tau_{g o}\right) \hat{a},
$$

where:

$$
\left\{\begin{array}{l}
\hat{C}_{h}=-f_{1} \phi_{11}-f_{2} \phi_{51}-f_{3} \phi_{61} \\
\hat{C}_{v}=-f_{1} \phi_{12}-f_{2} \phi_{52}-f_{3} \phi_{62} \\
\hat{C}_{a}=-f_{1} \phi_{13}-f_{2} \phi_{53}-f_{3} \phi_{63}
\end{array}\right.
$$

and:

$$
\begin{aligned}
f_{1}= & \frac{\phi_{54}\left(\tau_{g o}\right) \phi_{65}\left(\tau_{g o}\right)-\phi_{55}\left(\tau_{g o}\right) \phi_{64}\left(\tau_{g o}\right)}{\left|P_{2}\left(\tau_{g o}\right)\right|} \\
f_{2}=- & \frac{\phi_{14}\left(\tau_{g o}\right) \phi_{65}\left(\tau_{g o}\right)-\phi_{15}\left(\tau_{g o}\right) \phi_{64}\left(\tau_{g o}\right)}{\left|P_{2}\left(\tau_{g o}\right)\right|} \\
f_{3}= & \frac{\phi_{14}\left(\tau_{g o}\right) \phi_{55}\left(\tau_{g o}\right)-\phi_{15}\left(\tau_{g o}\right) \phi_{54}\left(\tau_{g o}\right)}{\left|P_{2}\left(\tau_{g o}\right)\right|} \\
\left|P_{2}\right|= & \phi_{14}\left(\phi_{55} \phi_{66}-\phi_{56} \phi_{65}\right)+\phi_{54}\left(\phi_{16} \phi_{65}-\phi_{15} \phi_{66}\right) \\
& +\phi_{64}\left(\phi_{15} \phi_{56}-\phi_{16} \phi_{55}\right)
\end{aligned}
$$

For brevity, argument $\tau_{g o}$ in $\phi_{i j}()$ and $P_{2}$ was omitted from Eqs. (30) and (32). Therefore, the normalized commanded acceleration can be obtained from Eqs. (14) and (29) as:

$$
\hat{u}(\tau)=-\hat{C}_{h}\left(\tau_{g o}, \hat{b}\right) \hat{h}-\hat{C}_{v}\left(\tau_{g o}, \hat{b}\right) \hat{v}-\hat{C}_{a}\left(\tau_{g o}, \hat{b}\right) \hat{a} .
$$

For the purpose of clarity, the constant $\hat{b}$ is added to the arguments of the normalized guidance gains. Hence, the optimal commanded acceleration is written in the following form:

$$
u(t)=A \hat{u}(\tau)=-C_{h} h-C_{v} v-C_{a} a,
$$

where the guidance gains, $C_{h}, C_{v}$, and $C_{a}$, are given by:

$$
\left\{\begin{array}{l}
C_{h}=\frac{1}{T^{2}} \hat{C}_{h} \\
C_{v}=\frac{1}{T} \hat{C}_{v} \\
C_{a}=\hat{C}_{a}
\end{array}\right.
$$

Similarly, the history of the commanded acceleration is obtained as $\left(\tau_{0}=0\right)$ :

$$
\hat{u}(\tau)=\left[-N_{1}(\tau)+N_{2}(\tau) P_{2}\left(\tau_{f}\right)^{-1} P_{1}\left(\tau_{f}\right)\right] \vec{x}(0),
$$

where:

$$
\begin{cases}N_{1}(\tau)=\left[\begin{array}{lll}
\phi_{61}(\tau) & \phi_{62}(\tau) & \phi_{63}(\tau)
\end{array}\right] \\
N_{2}(\tau)=\left[\begin{array}{lll}
\phi_{64}(\tau) & \phi_{65}(\tau) & \phi_{66}(\tau)
\end{array}\right]\end{cases}
$$

The behavior of the normalized guidance gains is shown in Figure 2 for different values of $\hat{b}=0.01,0.05,0.1$. As can be seen in the figure, the guidance gains reach the steady-state values when $\tau_{g o}$ approaches infinity, which, in practice, is for $\tau_{g o}=10 \sim 12$ for the mentioned values of weighting coefficients. These steady-state values are displayed in Figure 3 versus the normalized weighting coefficient $(\hat{b})$.

The steady state normalized guidance gains can also be obtained directly from the following algebraic Ricatti equation:

$$
0=-S A_{s}-A_{s}^{T} S-Q+S B R^{-1} B^{T} S,
$$

where $A_{s}$ is the system matrix of the state equation (6), $B=\left[\begin{array}{lll}0 & 0 & 1\end{array}\right]^{T}$ is the input matrix, $R=1$, and $Q$ is the weighting matrix of the state vector for a standard quadratic form:

$$
A_{s}=\left[\begin{array}{ccc}
0 & 1 & 0 \\
0 & 0 & 1 \\
0 & 0 & -1
\end{array}\right], \quad Q=\left[\begin{array}{ccc}
\hat{b} & 0 & 0 \\
0 & 0 & 0 \\
0 & 0 & 0
\end{array}\right]
$$

The costate vector is then given by $\vec{\lambda}(\tau)=S \vec{x}(\tau)$. Since only the third element of the costate, $\lambda_{a}$, is required for calculation of the commanded acceleration, only the third row of matrix $S$ is needed, that is:

$$
S_{31}=\sqrt{\hat{b}}
$$

$$
\begin{aligned}
& S_{32}=\frac{\sqrt{y_{1}}}{2}+\frac{\sqrt{-y_{1}+2 \sqrt{y_{1}^{2}+16 \hat{b}}}}{2}, \\
& S_{33}=\sqrt{1+2 S_{32}}-1,
\end{aligned}
$$




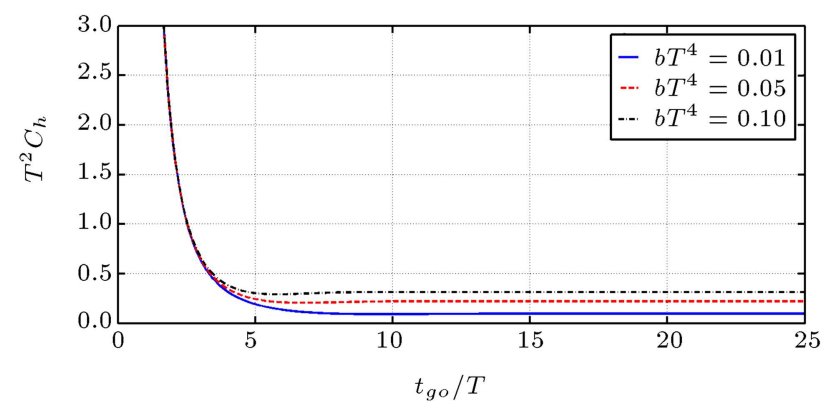

(a)

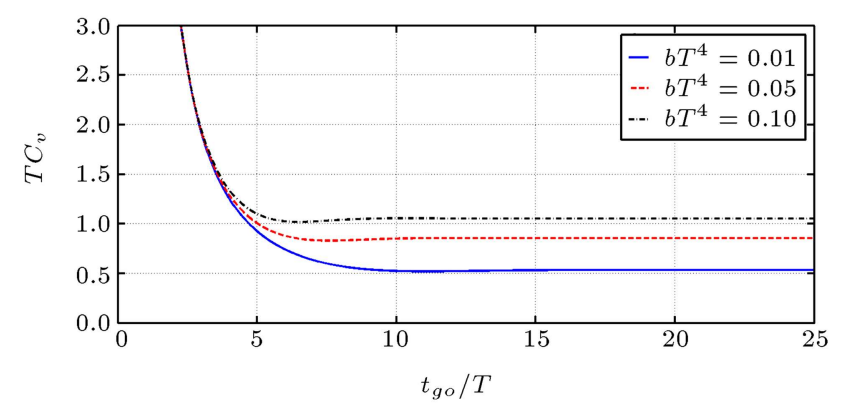

(b)

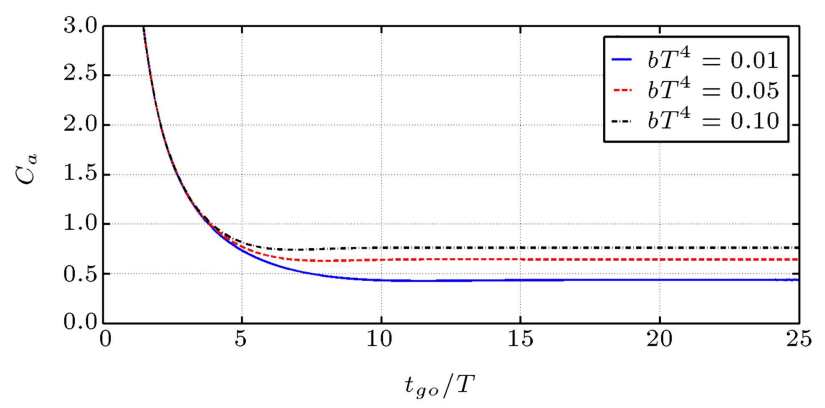

(c)

Figure 2. Normalized guidance gains profiles.

where:

$$
\begin{aligned}
& y_{1}=Z_{1}+Z_{2}, \\
& Z_{1,2}=\left(32 \hat{b}^{2} \pm \frac{32 \sqrt{12 \hat{b}^{3}+81 \hat{b}^{4}}}{9}\right)^{\frac{1}{3}} .
\end{aligned}
$$

The normalized commanded acceleration is then given by:

$$
\begin{aligned}
\hat{u}(\tau)= & -\hat{C}_{h}(\infty, \hat{b}) \hat{h}-\hat{C}_{v}(\infty, \hat{b}) \hat{v} \\
& -\hat{C}_{a}(\infty, \hat{b}) \hat{a}
\end{aligned}
$$

where:

$$
\begin{aligned}
& \hat{C}_{h}(\infty, \hat{b})=S_{31}, \\
& \hat{C}_{v}(\infty, \hat{b})=S_{32}, \\
& \hat{C}_{a}(\infty, \hat{b})=S_{33} .
\end{aligned}
$$

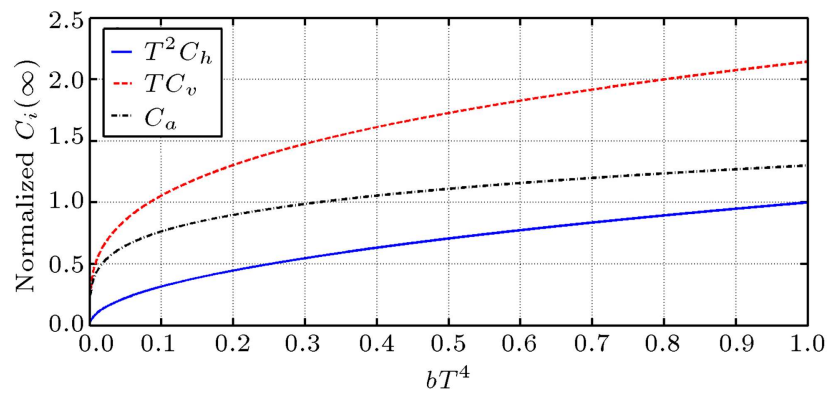

Figure 3. Steady-state normalized guidance gains vs. weighting coefficient.

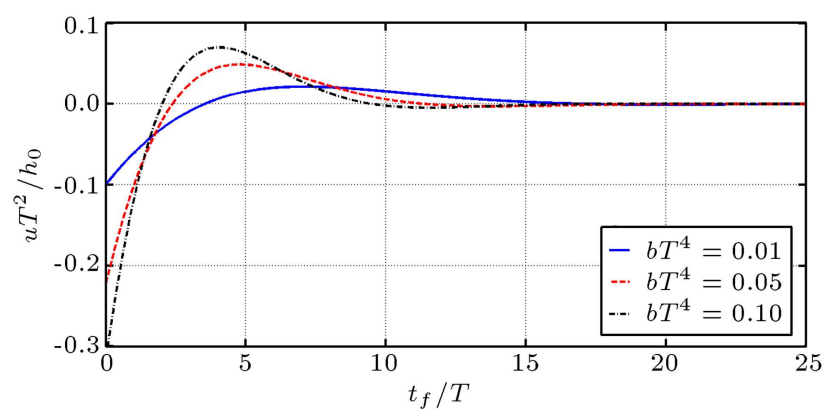

Figure 4. Normalized commanded acceleration vs. normalized time for different values of $\hat{b}=0.01,0.05,0.1$ $\left(v_{0}=0\right)$.

\section{Simulation results and discussions}

Here, the performance analysis of the First-Order Optimal Guidance (FOOG) strategy is investigated and compared with its steady-state solution and ZeroLag Optimal Guidance (ZLOG) law. First, consider a first-order transfer function described by Eq. (1) for the vehicle control system. The acceleration initial condition is set to zero. Three different normalizing factors are introduced for the problem, i.e. $A=h_{0} / T^{2}$, $A=v_{0} / T$, and $A=a_{\text {sat }}$, where $a_{\text {sat }}$ is the acceleration limit of the vehicle. Each normalizing factor is useful for a specific class of scenarios.

In the first class of scenarios, the initial vertical velocity is set to zero, i.e. $v_{0}=0$ without considering the acceleration limit. For this class, $A=h_{0} / T^{2}$ is chosen as the normalizing factor. Therefore, the behavior for the commanded acceleration and deviation from LOS can be solved numerically, once for all values of initial deviation from LOS and the control system time constant, as shown in Figures 4 and 5 . As expected, increasing the weighting factor increases the required acceleration commands, as depicted in Figure 4, resulting in a more rapid decrease in LOS deviation, as shown in Figure 5.

In the second class of scenarios, the initial deviation from LOS is assumed to be zero. In this class, $A=v_{0} / T$ may be chosen as a normalization factor. Similarly, increasing the weighting factor increases the required acceleration commands in order to decrease 


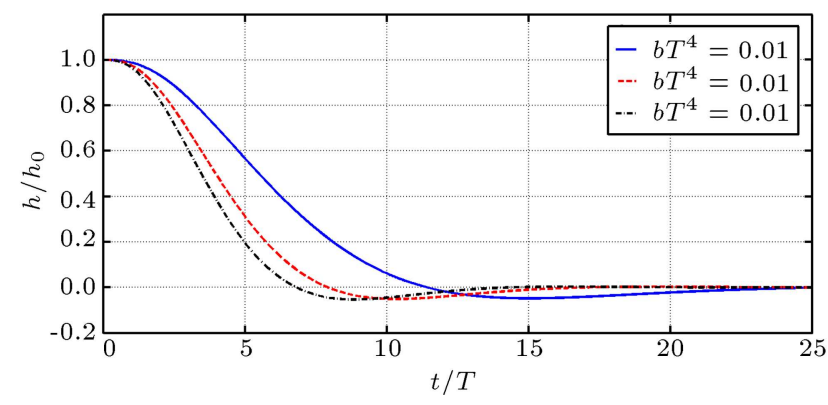

Figure 5. Normalized deviation vs. normalized time for different values of $\hat{b}=0.01,0.05,0.1\left(v_{0}=0\right)$.

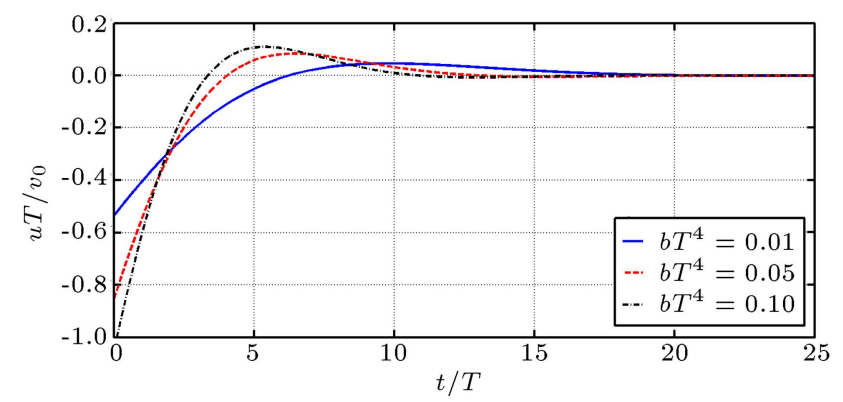

Figure 6. Normalized commanded acceleration vs. normalized time for different values of $\hat{b}=0.01,0.05,0.1$ $\left(h_{0}=0\right)$.

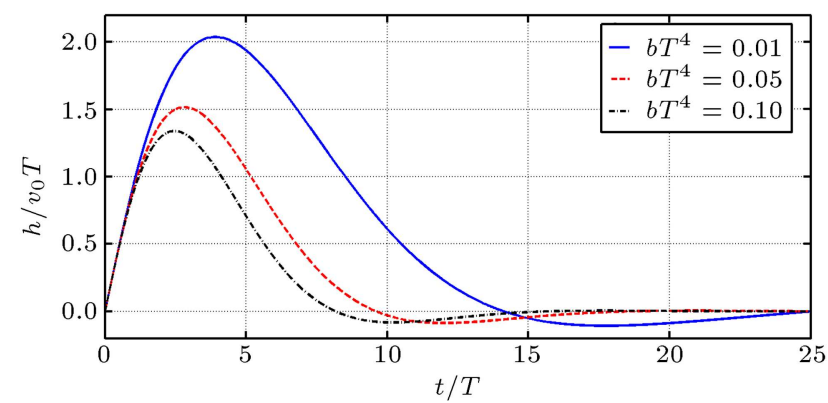

Figure 7. Normalized deviation vs. normalized time for different values of $\hat{b}=0.01,0.05,0.1\left(h_{0}=0\right)$.

the LOS deviation more rapidly, as shown in Figures 6 and 7. Therefore, there is a tradeoff to select an appropriate value for the weighting factor.

The mentioned normalization can be used for other situations, e.g. $\hat{h}_{0}=10$, as in Figures 8 and 9 , when $A=v_{0} / T$. We are now to compare the performance of the First-Order Optimal Guidance (FOOG), FOOG with steady-state gains labeled by FOOG $\left(t_{f}=\infty\right)$ in related figures, and the ZeroLag Optimal Guidance (ZLOG) in normalized form (Appendix B). The comparison of the three guidance laws is carried out for a second-order control system described by Eq. (7). The acceleration commands and LOS deviation are shown in Figures 8 and 9 , respectively, for the three guidance laws without acceleration limit, when $\hat{b}=0.01$ and $\hat{h}_{0}=10$. The acceleration commands for FOOG and FOOG $\left(t_{f}=\infty\right)$ are almost

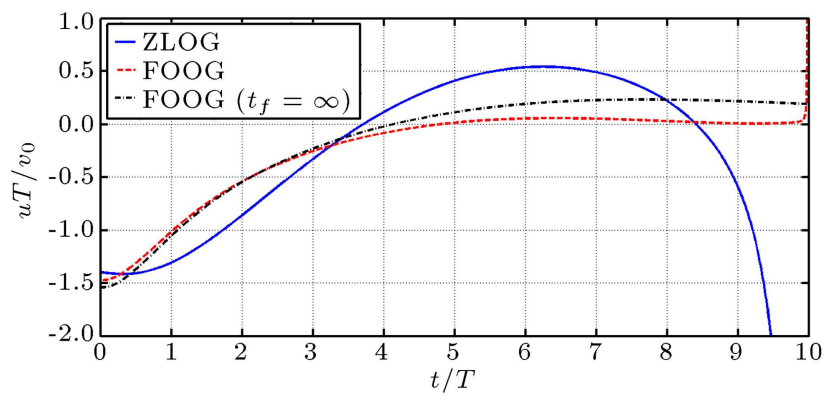

Figure 8. Normalized commanded acceleration vs. normalized time for three guidance laws $\left(h_{0} / v_{0} T=10\right.$, $\hat{b}=0.01)$.

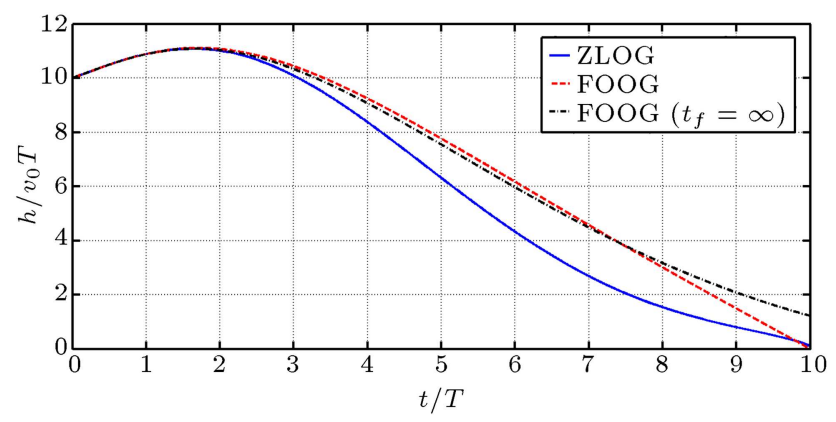

Figure 9. Normalized deviation vs. normalized time for three guidance laws $\left(h_{0} / v_{0} T=10, \hat{b}=0.01\right)$.

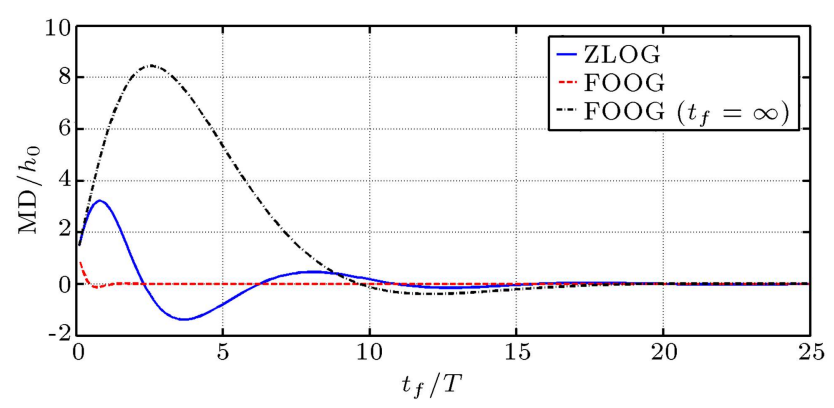

Figure 10. Normalized miss distance vs. normalized final time for three guidance laws $\left(v_{0} T / h_{0}=5, \hat{b}=0.05\right)$.

close together, as seen in Figure 8, but the acceleration commands for ZLOG are quite different, especially for the last instants (about the system time constant). Further simulations show that the differences between the performances of FOOG and its steady-state version increase for smaller $t_{f} / T$, and vice versa. The steadystate FOOG gives a similar performance, like FOOG for $\tau_{f}>20$. Figure 9 shows that FOOG $\left(t_{f}=\infty\right)$ produces more miss distances than those of the two other guidance laws.

To investigate more precisely, the normalized Miss Distances (MD) are compared for different total flight times in Figures 10 and 11 for different normalization factors, without an acceleration limit. The normalized miss distance of FOOG is smaller than that of the two others. In addition, as shown in Figure 12, the miss distance of zero-lag optimal guidance can be fluctuated 


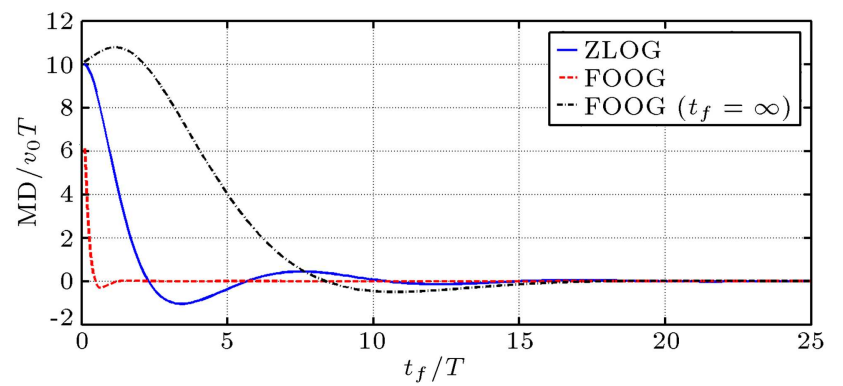

Figure 11. Normalized miss distance vs. normalized final time for three guidance laws $\left(h_{0} / v_{0} T=10, \hat{b}=0.05\right)$.

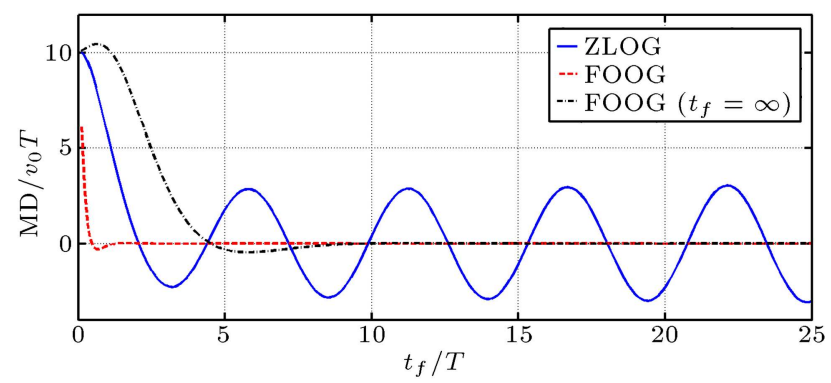

Figure 12. Normalized miss distance vs. normalized final time for three guidance laws $\left(h_{0} / v_{0} T=10, \hat{b}=0.8\right)$.

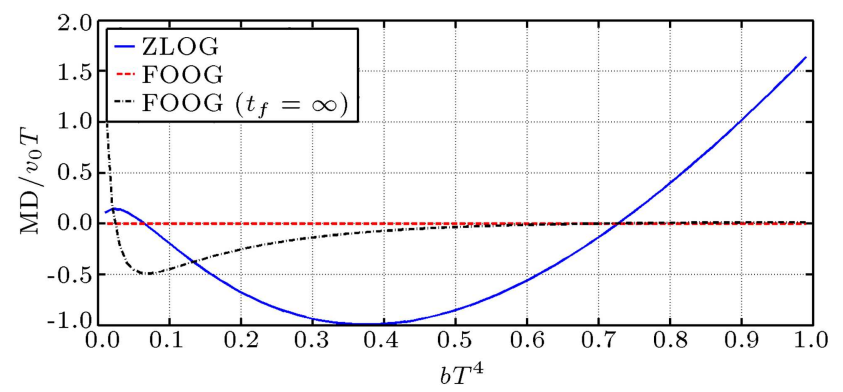

Figure 13. Normalized miss distance vs. normalized weighting factor $(\hat{b})$ for three guidance laws $\left(h_{0} / v_{0} T=10\right.$, $\left.\tau_{f}=10\right)$.

versus normalized final time, like an undamped oscillation, if large values of weighting factor are needed to satisfy the miss distance requirements. To investigate more precisely, the normalized miss distance is plotted versus the weighting factor in Figure 13 for $\tau_{f}=10$, showing the poor performance of the ZLOG when the weighting factor is increased. It is worth noting that point $\tau_{f}=10$ gives a relatively good result, with respect to other normalized final times for ZLOG, as shown in Figure 12.

Up to now, the performance analysis has been carried out with no acceleration limit. The effect of acceleration saturation is investigated here in Figures 14-17. The normalized miss distance for the three guidance laws is depicted in Figure 14 using the normalizing factor $A=a_{\text {sat }}$. The three guidance laws produce similar miss distances for small normalized flight time, depending on initial errors, because the

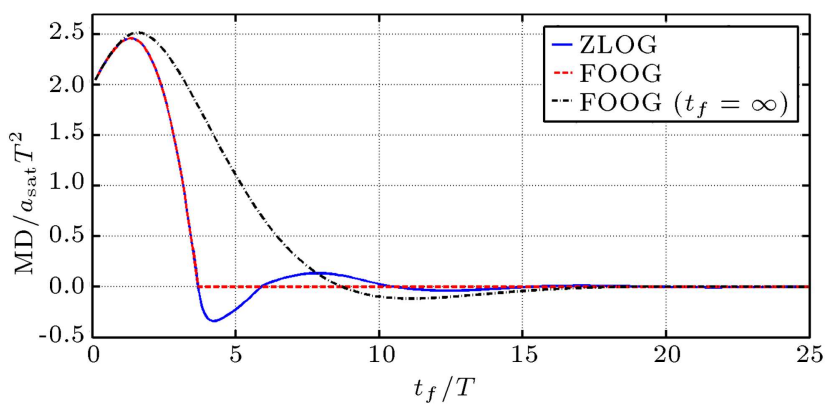

Figure 14. Normalized miss distance vs. normalized final time for three guidance laws $\left(h_{0} / a_{\text {sat }} T^{2}=2\right.$, $\left.v_{0} / a_{\text {sat }} T=0.5, \hat{b}=0.05\right)$.

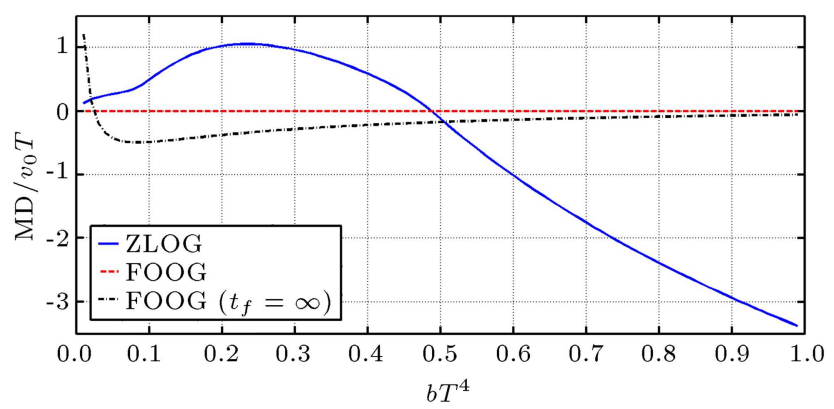

Figure 15. Normalized miss distance vs. normalized weighting factor $(\hat{b})$ for three guidance laws $\left(h_{0} / v_{0} T=10\right.$, $\left.\tau_{f}=10, a_{\text {sat }} T / v_{0}=2\right)$.

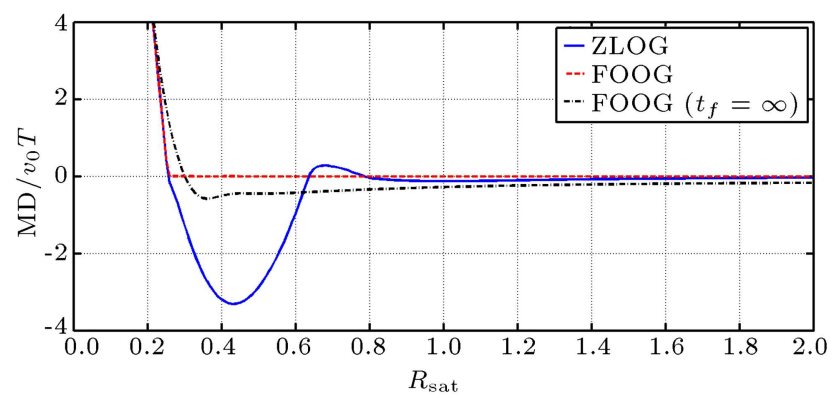

Figure 16. Normalized miss distance vs. normalized acceleration limit $\left(R_{\text {sat }}\right)$ for three guidance laws $\left(h_{0} / v_{0} T=10, \tau_{f}=15, \hat{b}=0.05\right)$.

accelerations of all three guidance strategies are saturated. If we have sufficient final time (or a larger acceleration limit), the acceleration comes off the limit and the performances of the guidance laws become different, resulting in a smaller miss distance for FOOG than the other two. For example, the miss distance at point $\tau_{f}=10$ is analyzed in Figure 15 versus weighting factor. This figure can be compared with the case without an acceleration limit, as shown before in Figure 13. The normalized miss distance versus normalized acceleration limit is depicted in Figure 16, for $\tau_{f}=15$ and $\hat{h}_{0}=10$. As seen in this figure, the performance of FOOG is superior to ZLOG in the middle range of showed normalized acceleration limit.

To investigate further the effect of weighting 


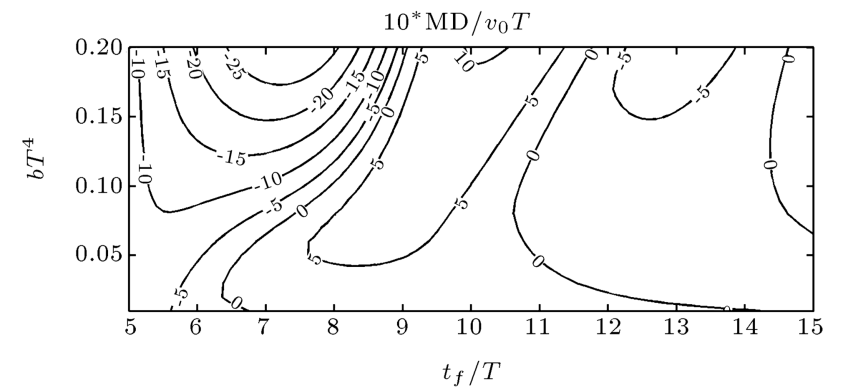

(a)

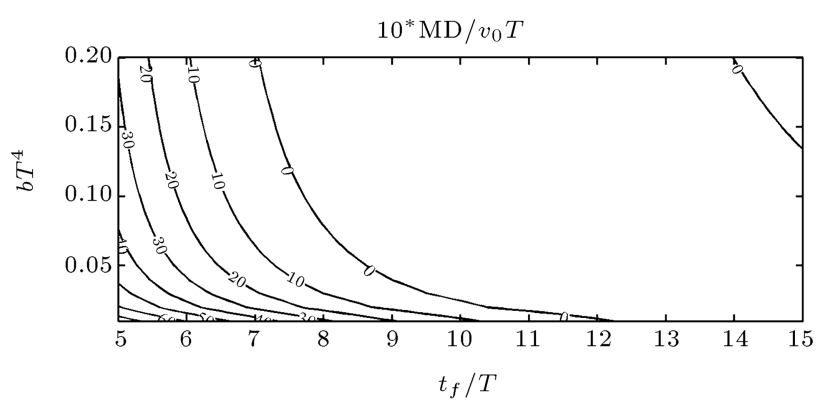

(b)

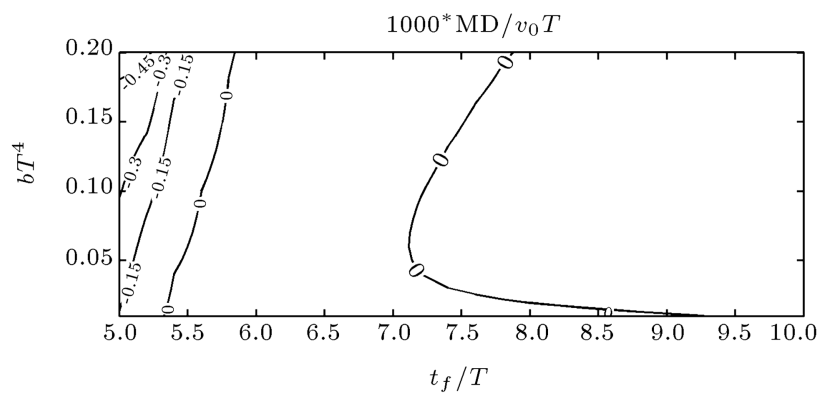

(c)

Figure 17. Normalized miss distance contours: normalized weighting factor vs. normalized final time for (a) ZLOG, (b) FOOG $\left(\tau_{f}=\infty\right.$ ), and (c) FOOG $\left(h_{0} / v_{0} T=10, a_{\text {sat }} T / v_{0}=2\right)$.

factor in miss distance, the normalized miss distance contours, constant $\mathrm{MD} / v_{0} T$, for the three guidance laws are plotted in Figure 17, when $h_{0} / v_{0} T=10$ and $a_{\text {sat }} T / v_{0}=2$. The figure is not shown for $t_{f} / T<5$ because the effect of the acceleration limit is dominant for prescribed initial errors and parameters. For ZLOG, a clear trend cannot be observed in Figure 17(a); however, for larger values of normalized final time, such a trend can be seen. The plotted contours for steady-state FOOG display a regular trend in Figure 17(b). Comparing Figure 17(a) and (b), shown for $10 \times \mathrm{MD} / v_{0} T$, determines in which regions the steady-state FOOG produce less miss distance than that of ZLOG. The normalized miss distance contour for FOOG is shown in Figure 17(c) plotted for $1000 \times \mathrm{MD} / v_{0} T$ to show better the very small values of normalized miss distance.

The allowable minimum final time to achieve a

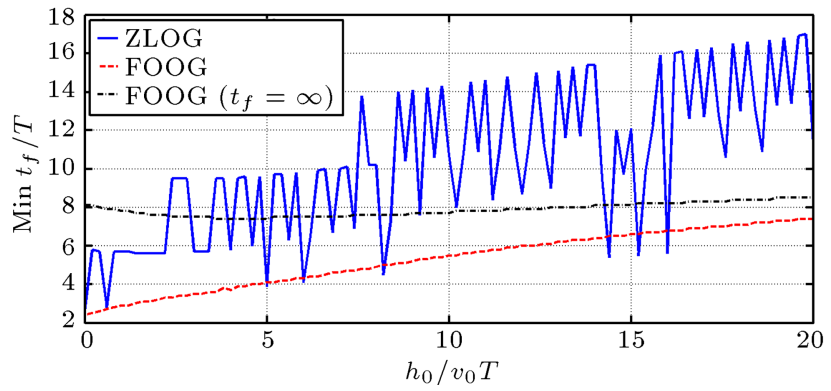

Figure 18. Normalized minimum final time vs. normalized initial distance to $\operatorname{LOS}\left(\hat{b}=0.1, a_{\text {sat }} T / v_{0}=2\right)$.

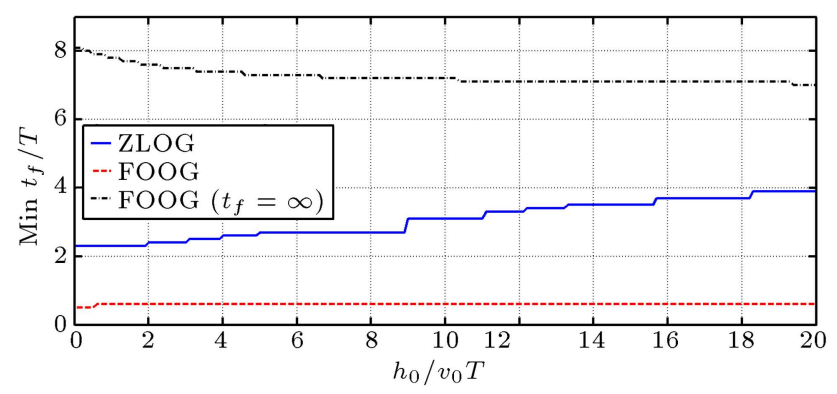

Figure 19. Normalized minimum final time vs. normalized initial distance to $\operatorname{LOS}\left(\hat{b}=0.1, a_{\text {sat }} T / v_{0}=5\right)$.

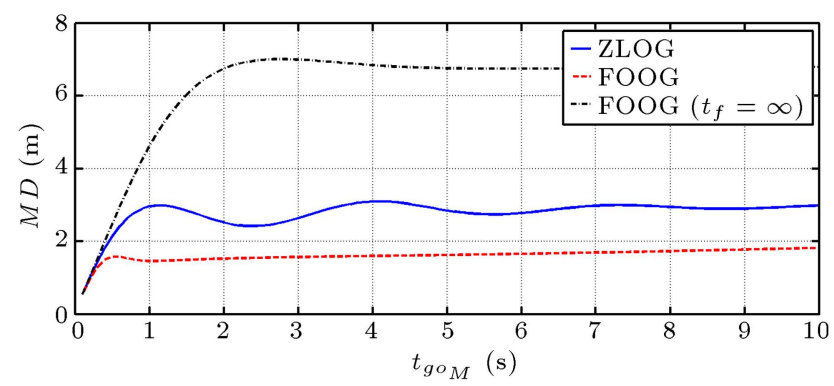

Figure 20. Miss distance vs. target maneuvering time to the final time for three guidance laws $(b=5, T=0.5$, $h_{0}=0, v_{0}=0, a_{\text {sat }}=10 g$ ).

predetermined miss distance is depicted in Figures 18 and 19 for the three guidance laws in normalized form. In these figures, the allowable normalized miss distance is chosen less than 0.02 , i.e. $\mathrm{MD} / v_{0} T<0.02$. As expected, the first-order optimal guidance law can work in smaller final times, compared to the others. The fluctuation in Figure 18 is due to an acceleration limit of $a_{\text {sat }} T / v_{0}=2$. Increasing the acceleration limit decreases the fluctuation, as seen in Figure 19.

Although the formulations have been developed in one dimension, they can be utilized in two dimensions (two channels), in practice. In addition, we are now to evaluate the performance of the three mentioned guidance laws in two-dimensional geometry for a special case of target maneuvers. In Figure 20, a stationary target moves from rest with a speed of $5 \mathrm{~m} / \mathrm{s}$ normal to the initial LOS in the last instants, and the vehicle having a speed of $250 \mathrm{~m} / \mathrm{s}$ launches without initial 
errors. The target maneuvering time to the final time is denoted by $t_{g o_{M}}$ in the figure. As shown in Figure 20, the miss distance of the first-order guidance law is less than the others for the mentioned case of maneuvering target.

\section{Conclusions}

An optimal three-point guidance law has been derived in a closed-loop for a first-order control system without acceleration limit. The final position and total flight time are both fixed. The optimal commanded acceleration has been developed in one dimension; however, it can be used in two channels separately in the same formulation. In the closed-loop formulation, the optimal gains are in terms of normalized timeto-go and normalized weighting factor; therefore, the time-to-go may be, in practice, estimated by vehicleto-target closing velocity and range-to-go. Moreover, the formulation is normalized in three forms to give normalized guidance gains and also normalized miss distance curves. Each of the normalizations has advantages for a normalized parametric study of the problem. The performance of the guidance laws is compared in normalized forms with zero-lag optimal guidance and a first-order optimal scheme with steady-state gains using a second-order control system with/without acceleration limit. The preliminary study shows that the miss distance of the first-order guidance law is smaller than the two mentioned schemes for small total flight times; however, a complete study with state estimation in the presence of noise and disturbance is suggested in a six-degree-of-freedom flight simulation.

\section{Nomenclature}

$\begin{array}{ll}a & \text { Vehicle acceleration } \\ A & \text { Normalizing parameter } \\ b & \text { Weighting coefficient } \\ C_{h}, C_{v}, C_{a} & \text { Guidance gains } \\ h & \text { Distance from line-of-sight } \\ H & \text { Hamiltonian } \\ \mathcal{L}^{-1} & \text { Inverse Laplace transform } \\ J & \text { Performance index } \\ s & \text { Laplace domain variable } \\ t_{f} & \text { Final time } \\ t_{g o} & \text { Time-to-go until the final time } \\ T & \text { Time constant } \\ u & \text { Commanded acceleration } \\ v & \text { Velocity component of vehicle normal } \\ \vec{x} & \text { to LOS } \\ \vec{\lambda} & \text { State vector } \\ & \text { Costate vector }\end{array}$

$d() / d \tau$

\section{References}

1. Shneydor, N.A., Missile Guidance and Pursuit: Kinematics, Dynamics and Control, Horwood Publishing, Chichester, England (1998).

2. Zarchan, P. "Tactical and strategic missile guidance", Progress in Astronautics and Aeronautics, 239, 6th Ed., AIAA (2012).

3. Lee, G.T. and Lee, J.G. "Improved command to lineof-sight for homing guidance", IEEE Transactions on Aerospace and Electronic Systems, 31(1), pp. 506-510 (1995).

4. Malaek, S.M. and Kosari, A.R. "Novel minimum time trajectory planning in terrain following flights", IEEE Transactions on Aerospace and Electronic Systems, 43(1), pp. 2-12 (2007).

5. Sujit, P.B., Saripalli, S. and Sousa, J.B. "Unmanned aerial vehicle path following, a survey and analysis of algorithms for fixed-wing UAVs", IEEE Control Systems Magazine, 34(1), pp. $42-59$ (2014).

6. Ha, I.J. and Chong, S. "Design of a CLOS guidance law via feedback linearization", IEEE Transactions on Aerospace and Electronic Systems, 28(1), pp. 51-63 (1992).

7. Arvan, M.R., and Moshiri, B. "Optimal fuzzy controller design for an anti-tank missile", Proc. of the International Conference on Intelligent and Cognitive Systems, Tehran, Iran, pp. 123-128 (1996).

8. Lin, C.M., Hsu, C.F. and Mon, Y.J. "Self-organizing fuzzy learning CLOS guidance law design", IEEE Transactions on Aerospace and Electronic Systems, 39(4), pp. 1144-1151 (2003).

9. Soleymani, T. and Saghafi, F. "Fuzzy trajectory tracking control of an autonomous air vehicle", The 2nd International Conference on Mechanical and Electronics Engineering (ICMEE), Kyoto, Japan (2010).

10. Yuan, L.Y. and Li, S.Y. "Missile guidance law design using nonlinear robust output regulation and TS model", The 2nd IEEE Conference on Industrial Electronics and Applications, ICIEA 2007, Harbin, China (2007).

11. Fiorentini, L. and Serrani, A. "Adaptive restricted trajectory tracking for a non-minimum phase hypersonicvehicle model", Automatica, 48(7), pp. 1248-1261 (2012).

12. Benshabat, D.G. and Bar-Gill, A. "Robust commandto-line-of-sight guidance via variable-structure control", IEEE Transactions on Control Systems Technology, 3(3), pp. 356-361 (1995). 
13. Yang, K. "Path following control performance comparison for an rotary wing unmanned aerial vehicle", 10th International Conference on Ubiquitous Robots and Ambient Intelligence (URAI), Ramada Plaza Jeju Hotel, Jeju, Korea (Oct. 31-Nov. 2, 2013).

14. Pourtakdoust, S.H. and Nobahari, H. "Line-of-sight guidance law optimization for ground-to-air missiles", First Conference of Aerospace industries Organization, Tehran, Iran (2000).

15. Ratnoo, A., Sujit, P.B. and Kothari, M. "Adaptive optimal path following for high wind flights", in Proc. of 18th International Federation of Automatic Control (IFAC) World Congress, Milan, Italy, pp. 12,98512,990 (Aug. 28-Sept. 2, 2011).

16. Wang, J. and Xin, M. "Integrated optimal formation control of multipleunmanned aerial vehicles", IEEE Transactions on Control Systems Technology, 21(5), pp. 1731-1744 (2013).

17. Guo, J., et al. "Design of automatic steering controller for trajectory tracking of unmanned vehicles using genetic algorithms", IEEE Transactions on Vehicular Technology, 61(7), pp. 2913-2924 (2012).

18. Nobahari, H. and Pourtakdoust, S.H. "An optimal fuzzy two-phase CLOS guidance law design using ant colony optimization", The Aeronautical Journal, 111(1124), pp. 621-636 (2007).

19. Nodland, D., Zargarzadeh, H. and Jagannathan, S. "Neural network-based optimal adaptive output feedback control of a helicopter UAV", IEEE Transactions on Neural Networks and Learning Systems, 24(7), pp. 1061-1073 (2013).

20. Rusnak, I. and Meir, L. "Modern guidance law for high order autopilot", Journal of Guidance Control, and Dynamics, 14(5), pp. 1056-1058 (1991).

21. Jalali-Naini, S.H. and Esfahanian, V. "Closed-form solution of line-of-sight trajectory for nonmaneuvering targets", AIAA Journal of Guidance, Control, and Dynamics, 23(2), pp. 365-366 (2000).

22. Jalali-Naini, S.H. and Esfahanian, V. "Solution of three-dimensional line-of-sight guidance with a moving tracker", Scientia Iranica, 11(1\&2), pp. 138-145 (2004).

23. Balakrishnan, S.N., Tsourdos, A. and White, B.A., Advances in Missile Guidance, Control, and Estimation, Automation and Control Engineering, CRC Press, Taylor \& Francis Group, NW (2013).

24. Kain, J.E. and Yost, D.J. "Command to Line-ofsight guidance: a stochastic optimal control problem", Journal of Spacecraft and Rocket, 14(7), pp. 438-444 (1977).

25. Jalali-Naini, S.H. and Sajjadi, S.H. "Closed-loop optimal guidance for first-order control system", The 11th Iranian Aerospace Society Conference, Tehran, Iran (2012).

26. Jalali-Naini, S.H. "Normalized miss distance analysis of single-lag optimal guidance law with radome effect, saturation and fifth-order control system", Scientia Iranica, Transactions B, 21(5), pp. 1683-1692 (2014).
27. Bryson, A.E. and Ho, Y.C., Applied Optimal Control, Hemisphere Publishing Corporation, Washington, D.C. (1975).

28. Spiegel, M.R., Lipschutz, S. and Liu, J., Mathematical Handbook of Formulas and Tables, Schaum's Outline Series, 3rd Ed., McGraw-Hill, NY (2009).

\section{Appendix A: Derivation of state transition matrix}

The state transition matrix for system matrix (19) is obtained by Eq. (22). For calculation of the state transition matrix, first, the characteristic equation and its roots are obtained. The characteristic equation is found to be:

$$
\left|s I-A_{p}\right|=s^{6}-s^{4}-\hat{b}=0 .
$$

The roots of the characteristic equation can be found by the general solution of a cubic algebraic equation [28]. Therefore, we have:

$$
X^{3}-X^{2}-\hat{b}=0,
$$

where $X=s^{2}$. According to [28], when $D=Q_{1}^{3}+Q_{2}^{2}>$ 0 , we have one real and one conjugate pair of roots:

$$
Q_{1}=-\frac{1}{9}, \quad Q_{2}=\frac{1}{27}+\frac{\hat{b}}{2} \Rightarrow D=\frac{\hat{b}}{27}+\frac{\hat{b}^{2}}{4}>0 .
$$

As seen in the preceding inequality, $D$ is positive when $\hat{b}>0$. In this case, the characteristic equation (A.1) can be factorized in the following form:

$$
\left(s^{2}-\alpha^{2}\right)\left(s^{2}+2 \zeta \omega s+\omega^{2}\right)\left(s^{2}-2 \zeta \omega s+\omega^{2}\right) .
$$

The real root of Eq. (A.2) is represented by $\alpha^{2}$, and is given by:

$$
\alpha=\sqrt{Z_{1}+Z_{2}+\frac{1}{3}}
$$

where:

$$
Z_{1,2}=\left(Q_{2} \pm \sqrt{D}\right)^{\frac{1}{3}}=\left(\frac{1}{27}+\frac{\hat{b}}{2} \pm \frac{\sqrt{12 \hat{b}+81 \hat{b}^{2}}}{18}\right)^{\frac{1}{3}}
$$

After some manipulation, $\omega$ and $\zeta$ are obtained as:

$$
\begin{aligned}
& \omega=\left(\frac{\hat{b}}{\alpha^{2}}\right)^{1 / 4} \\
& \zeta=\frac{1}{\sqrt{2}}\left(1-\frac{\alpha^{2}-1}{2 \omega^{2}}\right)^{1 / 2} .
\end{aligned}
$$

The behavior of $\alpha, \omega$ and $\zeta$ versus $\hat{b}$ can be viewed in Figure A.1. It can be shown that the expressions under 


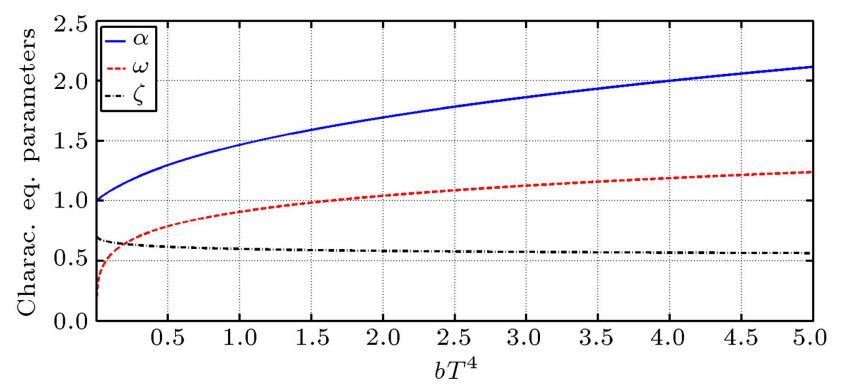

Figure A.1. The behavior of $\alpha, \omega$ and $\zeta$ vs. $\hat{b}=b T^{4}$.

the radical sign in Eqs. (A.5) and (A.8) are positive for our problem $(\hat{b}>0)$ and also $\zeta^{2}<1 / 2$.

From Eq. (22), the state transition matrix can be written as:

$$
\Phi(s)=\frac{M_{1} s^{5}+M_{2} s^{4}+M_{3} s^{3}+M_{4} s^{2}+M_{5} s+M_{6}}{s^{6}-s^{4}-\hat{b}},
$$

where:

$$
\left\{\begin{array}{l}
M_{1}=I_{6 \times 6} \\
M_{2}=A_{p} \\
M_{3}=A_{p}^{2}-I \\
M_{4}=A_{p}\left(A_{p}^{2}-I\right) \\
M_{5}=A_{p}^{2}\left(A_{p}^{2}-I\right) \\
M_{6}=A_{p}^{3}\left(A_{p}^{2}-I\right)
\end{array}\right.
$$

Using Eq. (A.4), the partial fraction form of the state transition matrix may be written as in Eq. (A.11) as shown in Box I.

Using the following Laplace transform formulas $\left(\omega>0, \zeta^{2}<1\right)$ :

$\Phi_{r}(t)=\mathcal{L}^{-1}\left\{\frac{C_{1} s+C_{2}}{s^{2}-\alpha^{2}}\right\}=C_{1} \cosh (\alpha t)+\frac{C_{2} \sinh (\alpha t)}{\alpha(\mathrm{A} .18)}$

$$
\begin{aligned}
\Phi_{p c}(t) & =\mathcal{L}^{-1}\left\{\frac{C_{3} s+C_{4}}{s^{2}+2 \zeta \omega s+\omega^{2}}\right\} \\
= & e^{-\zeta \omega t}\left(C_{3} \cos \left(\omega_{d} t\right)+\frac{\left(C_{4}-C_{3} \zeta \omega\right) \sin \left(\omega_{d} t\right)}{\omega_{d}}\right)
\end{aligned}
$$

$\Phi_{n c}(t)=\mathcal{L}^{-1}\left\{\frac{C_{5} s+C_{6}}{s^{2}-2 \zeta \omega s+\omega^{2}}\right\}$$$
=e^{\zeta \omega t}\left(C_{5} \cos \left(\omega_{d} t\right)+\frac{\left(C_{6}+C_{5} \zeta \omega\right) \sin \left(\omega_{d} t\right)}{\omega_{d}}\right),
$$

where:

$$
\omega_{d}=\omega \sqrt{1-\zeta^{2}}
$$

The state transition matrix is finally obtained as follows:

$$
\Phi(t)=\Phi_{r}(t)+\Phi_{p c}(t)+\Phi_{n c}(t) .
$$

\section{Appendix B: Normalized perfect LOS guidance}

The optimal guidance solution to minimize performance index (10), subject to the differential equation constraint, $\ddot{h}=a$, and the initial conditions, $h(0)=0$, $v(0)=0$, and the final conditions, $h\left(t_{f}\right)=0$ and $v\left(t_{f}\right)=$ free, is obtained as follows [14]:

$$
u(\tau)=-C_{h}\left(t_{g o}, b\right) h-C_{v}\left(t_{g o}, b\right) v
$$

where:

$$
\begin{aligned}
& C_{h}= \\
& -\frac{2 \beta^{2}\left[\sin \left(\beta t_{g o}\right) \cos \left(\beta t_{g o}\right)+\sinh \left(\beta t_{g o}\right) \cosh \left(\beta t_{g o}\right)\right]}{\sin \left(\beta t_{g o}\right) \cos \left(\beta t_{g o}\right)-\sinh \left(\beta t_{g o}\right) \cosh \left(\beta t_{g o}\right)(\mathrm{B} .2 \mathrm{a})} \\
& C_{v}= \\
& -\frac{2 \beta\left[\sin ^{2}\left(\beta t_{g o}\right)+\sinh ^{2}\left(\beta t_{g o}\right)\right]}{\sin \left(\beta t_{g o}\right) \cos \left(\beta t_{g o}\right)-\sinh \left(\beta t_{g o}\right) \cosh \left(\beta t_{g o}\right)}, \quad(\mathrm{B} .2 \mathrm{~b}) \\
& \beta=b^{\frac{1}{4}} / \sqrt{2} .
\end{aligned}
$$

Although the preceding commanded acceleration is optimal for a perfect control system, the commanded acceleration should be normalized for our performance analysis when the control system is taken as a secondorder transfer function, that is:

$$
\hat{u}(\tau)=-\hat{C}_{h}\left(\tau_{g o}, \hat{b}\right) \hat{h}-\hat{C}_{v}\left(\tau_{g o}, \hat{b}\right) \hat{v}
$$

where:

$$
\begin{aligned}
& \hat{C}_{h}= \\
& -\frac{2 \hat{\beta}^{2}\left[\sin \left(\hat{\beta} \tau_{g o}\right) \cos \left(\hat{\beta} \tau_{g o}\right)+\sinh \left(\hat{\beta} \tau_{g o}\right) \cosh \left(\hat{\beta} \tau_{g o}\right)\right]}{\sin \left(\hat{\beta} \tau_{g o}\right) \cos \left(\hat{\beta} \tau_{g o}\right)-\sinh \left(\hat{\beta} \tau_{g o}\right) \cosh \left(\hat{\beta} \tau_{g o}\right)(\mathrm{B} .4 \mathrm{a})}, \\
& \hat{C}_{v}= \\
& -\frac{2 \hat{\beta}\left[\sin ^{2}\left(\hat{\beta} \tau_{g o}\right)+\sinh ^{2}\left(\hat{\beta} \tau_{g o}\right)\right]}{\sin \left(\hat{\beta} \tau_{g o}\right) \cos \left(\hat{\beta} \tau_{g o}\right)-\sinh \left(\hat{\beta} \tau_{g o}\right) \cosh \left(\hat{\beta} \tau_{g o}\right)(\mathrm{B} .4 \mathrm{~b})} \\
& \hat{\beta}=T \beta=\hat{b}^{\frac{1}{4}} / \sqrt{2} .
\end{aligned}
$$

The normalized commanded acceleration and guidance gains are defined as before, i.e., similar to Eqs. (33)(35). 


$$
\frac{M_{1} s^{5}+M_{2} s^{4}+M_{3} s^{3}+M_{4} s^{2}+M_{5} s+M_{6}}{s^{6}-s^{4}-\hat{b}}=\frac{C_{1} s+C_{2}}{s^{2}-\alpha^{2}}+\frac{C_{3} s+C_{4}}{s^{2}+2 \zeta \omega s+\omega^{2}}+\frac{C_{5} s+C_{6}}{s^{2}-2 \zeta \omega s+\omega^{2}},
$$

where:

$$
\begin{aligned}
C_{1}= & \frac{\alpha^{2} M_{1}+\alpha M_{3}+M_{5}}{2 \omega^{4}+\alpha^{4}}, \\
C_{2}= & \frac{\alpha^{2} M_{2}+\alpha M_{4}+M_{6}}{2 \omega^{4}+\alpha^{4}}, \\
C_{3}= & \frac{1}{4} \frac{4 \zeta \omega^{7} M_{1}+\left(\alpha^{2} \omega^{4}-2 \omega^{6}\right) M_{2}-2 \alpha^{2} \zeta \omega^{3} M_{3}}{\omega^{3} \zeta\left(2 \omega^{4}+\alpha^{4}\right)}+\frac{1}{4} \frac{\left(\omega^{4}+\alpha^{2} \omega^{2}\right) M_{4}-2 \zeta \omega^{3} M_{5}+\left(4 \zeta^{2} \omega^{2}-\omega^{2}-\alpha^{2}\right) M_{6}}{\omega^{3} \zeta\left(2 \omega^{4}+\alpha^{4}\right)} \\
C_{4}= & \frac{1}{4} \frac{\left(2 \omega^{8}-\alpha^{2} \omega^{6}\right) M_{1}+2 \alpha^{2} \zeta \omega^{5} M_{2}-\left(\omega^{6}+\alpha^{2} \omega^{4}\right) M_{3}+2 \zeta \omega^{5} M_{4}}{\omega^{3} \zeta\left(2 \omega^{4}+\alpha^{4}\right)} \\
& +\frac{1}{4} \frac{\left(\omega^{4}-4 \zeta^{2} \omega^{4}+\alpha^{2} \omega^{2}\right) M_{5}+2 \zeta \omega\left(4 \zeta^{2} \omega^{2}-2 \omega^{2}-\alpha^{2}\right) M_{6}}{\omega^{3} \zeta\left(2 \omega^{4}+\alpha^{4}\right)} \\
C_{5}= & \frac{1}{4} \frac{4 \zeta \omega^{7} M_{1}+\left(2 \omega^{6}-\alpha^{2} \omega^{4}\right) M_{2}-2 \alpha^{2} \zeta \omega^{3} M_{3}-\left(\omega^{4}+\alpha^{2} \omega^{2}\right) M_{4}}{\omega^{3} \zeta\left(2 \omega^{4}+\alpha^{4}\right)}+\frac{1}{4} \frac{-2 \zeta \omega^{3} M_{5}+\left(\omega^{2}-4 \zeta^{2} \omega^{2}+\alpha^{2}\right) M_{6}}{\omega^{3} \zeta\left(2 \omega^{4}+\alpha^{4}\right)} \\
C_{6}= & \frac{1}{4} \frac{\left(\alpha^{2} \omega^{6}-2 \omega^{8}\right) M_{1}+2 \alpha^{2} \zeta \omega^{5} M_{2}+\left(\omega^{6}+\alpha^{2} \omega^{4}\right) M_{3}+2 \zeta \omega^{5} M_{4}}{\omega^{3} \zeta\left(2 \omega^{4}+\alpha^{4}\right)} \\
& +\frac{1}{4} \frac{\left(4 \zeta^{2} \omega^{4}-\omega^{4}-\alpha^{2} \omega^{2}\right) M_{5}+2 \zeta \omega\left(4 \zeta^{2} \omega^{2}-2 \omega^{2}-\alpha^{2}\right) M_{6}}{\omega^{3} \zeta\left(2 \omega^{4}+\alpha^{4}\right)}
\end{aligned}
$$

Box I

\section{Bioghraphies}

Seyed Hamid Jalali-Naini received his BS and MS degrees in Mechanical Engineering from Amirkabir University of Technology, Tehran, Iran, and the University of Tehran, Iran, in 1994 and 1996, respectively, and a PhD degree in Aerospace Engineering from Sharif University of Technology, Tehran, Iran, in 2008. He is currently Assistant Professor of Aerospace Engineering at Tarbiat Modares University, Iran. His research interests are in the area of guidance and control of aerospace vehicles.

Sayyed Hesam Sajjadi received his BS degree in Aerospace Engineering, in 2008, from Sharif University of Technology, Tehran, Iran, and his MS degree, in 2010, from Tarbiat Modares University, Iran, where he is presently pursuing his $\mathrm{PhD}$ degree. His research interests include optimal guidance and control, trajectory tracking, and system integration. 